\title{
Design of an educational framework in introducing an unknown food crop into a farm worker community for ensuring food security
}

\author{
Innike Rajput ${ }^{1 *}$, Hettie C Schönfeldt ${ }^{2}$ and Rozanne Kruger ${ }^{1,3}$ \\ ${ }^{1}$ Department of Consumer Science, Faculty of Natural and Agricultural Sciences, University of Pretoria, 0028, Pretoria, \\ South Africa. \\ ${ }^{2}$ Department of Animal and Wildlife Sciences, Faculty of Natural and Agricultural Sciences, University of Pretoria, P.O. Box \\ 363, Silverton, 0127, Pretoria, South Africa. \\ ${ }^{3}$ Institute of Food, Nutrition and Human Health, Massey University, Albany, Private Bag102904, North Shore, Auckland, \\ 0745, New Zealand. \\ Accepted 17 May, 2012
}

This study aims to design an educational framework based on the utilization cycle as a food-based strategy, integrating both agricultural and nutritional components, and also to empower people to improve their food security by introducing an unknown food crop into a specific community based on an in-depth understanding of current practices using known food crops. It was carried out in a rural farm-worker community in the Free State Province of South Africa. An educational framework was designed to guide the nutrition intervention strategy. This strategy was based on information gathered on the utilization of spinach (known crop) and was then applied to design a training manual for the intervention using an unknown crop (orange-fleshed sweet potato) in collaboration with agricultural extension officers. The community was explored at grass-roots level, and the current utilization patterns of spinach were observed. Behaviours, habits and drivers behind utilization was identified and combined with theory to design the educational framework for a food-based intervention. The framework was tested and refined by exposing agricultural officers to it. The design of the strategy is a problem-solution approach, which consists of 4 phases: (1) addressing shortcomings of current diet not providing the minimum daily requirements for various vitamins and minerals as per the findings of the NFCS, (2) addressing the main underlying causes - which are low consumption of fruits and vegetables, and low dietary diversity, (3) proposed changes - inclusion of OFSP in their diets to not only increase dietary diversity but to also improve the status of vitamin A, and (4) desired outcome optimal utilization of OFSP through home garden cultivation. By observing the current strategies applied to the utilization of food crops, a framework was designed suitable to the unique local situation of a community to ensure maximum acceptance and application of unknown food crops. By involving agricultural extension officers in the process, the strategy was refined to be used by trainers in any rural community to implement introduction of new food crops to support the community in utilizing the new food crop and to ultimately improve dietary diversity.

Key words: Food-based approach, home-gardens, utilization cycle, educational framework, agricultural extension officers, rural farm worker community.

\section{INTRODUCTION}

Food-based approaches are currently being utilized in

*Corresponding author. E-mail: innike@gmail.com. Tel: +27 833989639. many studies in many parts of the world to alleviate food insecurity and malnutrition. Currently, there is an abundance of agriculture-based frameworks available. However, these are not focused on nutrition information or dietary behaviours (Morris et al., 2002). At the time of 
this study, no widely tested curriculum could be found that links nutrition lessons to growing vegetables in the garden (agriculture) especially, if new food crops are introduced (Faber and Van Jaarsveld, 2007).

The National Food Consumption Survey (NFCS), conducted during 1999 showed a monotonous diet with a lack of intake of nutrients such as vitamin $A$, iron and iodine in the South African diet, especially, amongst children (Labadarios, 2002). The national prevalence of vitamin A deficiency among 1 to 9 years-old children in South Africa was shown to be $64 \%$ (Labadarios, 2002). As part of a food-based approach, this could be alleviated by the increase in consumption of vitamin $A$ rich fruits and vegetables. According to Heim et al. (2011), the home food environment may be the most prominent environmental factor influencing a child's eating behaviour as home availability and accessibility to fruits and vegetables amongst other things have been associated with children's fruit and vegetable intake. There is a worldwide concession that a link exists between poor nutrition and food choices (Zeidan, 2007).

\section{Support for food-based strategies}

The most effective and sustainable way to overcome vitamin A deficiency is through food-based strategies (Underwood, 2000). This can be achieved by dietary diversification (food supplementation, horticultural interventions, management of proper distribution, and availability of vitamin A rich foods), nutrition education on all issues related to vitamin $A$ (for example, sources of vitamin $A$, importance of and need for vitamin $A$, methods of obtaining vitamin A rich foods, and community involvement and participation in the process), and food fortification (identification of foods that can be fortified, development of proper cost-effective methods taken into account, local food tastes and availability, and development of a mechanism to reach the most needy) (Chakravarty, 2000).

Food sources of vitamin A or pro-vitamin A (Bcarotene) that are new in the local food supply must be introduced with education or media techniques in ways that make the foods culturally acceptable to both the community and the individual (Kuhnlein, 2000). Kuhnlein (2000) also states that identification of the food resources, their methods of preservation, and preparation for consumption, as well as, knowledge of the amounts of the food consumed by vulnerable groups must be recorded for their potential contribution to food-based programs. However, introducing people to new crops and/or new food products does not necessarily result in their adoption to cultivation and/or consumption. Low et al. (2007) states that food based approaches have been found to have a promising role in integrated strategies, but that many knowledge gaps still exist, specifically on efficacy, effectiveness, cost effectiveness and sustainability. A food-to-food fortification approach assumes that there are locally available naturally rich food sources of vitamin A that are currently being underutilized, and it encourages those preparing meals to incorporate these foods into their vitamin A poor diets (Underwood, 2000). To some, food-based strategies suggest only homestead gardens, which, they argue, are not a sustainable intervention for micronutrient control on a national scale. Food-based strategies should be viewed as encompassing the total food chain from production in gardens or fields, to procurement from local or centralized markets, to processing domestically in preparation of meals or commercially, to consumption within or outside the home (Underwood, 2000).

\section{Successes and failures of food-gardens}

A small-scale pilot study by Faber et al. (2002) aimed at increasing the production of yellow and dark-green leafy vegetables in order to increase the serum retinol concentrations of 2 to 5-years-old children in South Africa was developed as an integrated health and gardening system and adapted to local conditions and gardening practices, thus, making the model viable in the rural African context. The children's caregivers were trained by nutrition monitors. The nutritional education component of the food production program focused on the relation between vitamin $A$ and health, the identification of vitamin A rich foods, cooking methods, and the importance of a home garden as source of vitamin A rich foods. The results were that twenty months after implementation of the home-gardening program, approximately, one-third of all households in the experimental village had a project garden.

Maternal knowledge regarding vitamin A nutrition, the frequency of consumption of yellow and dark-green leafy vegetables, and the serum retinol concentrations of 2 to 5 -years-old children were more favorable in the experimental village than in the control village. The home-gardening program strengthened existing gardening activities, and it was recommended that the yellow and dark-green leafy vegetables be planted in addition to existing crops, thereby, increasing the variety of vegetables available (Faber et al., 2002).

Low et al. (2007) designed a study with the aim to assess whether an integrated package of agriculture, nutrition, and market interventions focused on introduction and promotion of orange-fleshed sweet potato (OFSP) could increase vitamin A intake and serum retinol concentrations in young children in Mozambique. The new varieties were well accepted by farmers and consumers, including targeted children. In intervention areas, mean sweet potato plot sizes were nearly 10 times higher and OFSP emerged as the least expensive source of vitamin A in local markets. Nutrition knowledge increased in both men and women in the intervention area. For intervention children, the $24 \mathrm{~h}$ recall data 
suggested that OFSP was a major source of vitamin A, providing $90 \%$ of vitamin A for those children who consumed it the previous day and $35 \%$ for intervention children overall. Median vitamin A intakes were much higher for children in intervention areas compared with controls. OFSP was eaten regularly by intervention children during harvest season. Even gardens implemented at schools have shown very positive results. In a study by McAleese and Rankin (2007), gardenbased activities along with nutrition education resulted in significant increases in fruit and vegetable consumption amongst school children. In addition, vitamins $\mathrm{A}$ and $\mathrm{C}$, and fibre intakes showed significant increases. These results help to show the importance of hands-on activities when attempting to change nutrition-related behaviour such as fruit and vegetable consumption, building selfefficacy regarding the preparation of fruits or vegetables, increasing nutrition knowledge and awareness. Home gardening has multiple benefits, and many studies focus on narrow achievements only and fail to account for other successes (Niñez, 1984; Von Braun, 1993). If all benefits are considered, the benefit:cost ratio of gardening projects will likely be more favourable than alternative interventions. Dietary supplements and fortified foods have their place in clinical management, but their overall efficacy due to costs and the ability of poor communities to sustain fortification and supplementation programs, remains highly debatable (Zotor and Amuna, 2008). With fortification, there are limits to the amount of some micronutrients that can be added to a foodstuff due to their cost and their effect on the sensory properties of the food (Allen, 2008). Fortification of home-produced foods is difficult, and as such, limits the potential reach of this strategy to the most needy of the population. Compliance with supplementation programs is notoriously poor, and apart from some specific situations (such as supplementation of pregnant and lactating women, or children in school), these programs have been relatively ineffective. Supplementation also increases risk of micronutrient interactions and adverse effects (Allen, 2008).

There are advantages in utilising commonly-accessible affordable identifiable food sources and processing methods that are familiar and culturally appropriate to poor communities. There is also evidence that natural food sources of nutrients are better absorbed than expensive synthetic supplements (Zotor and Amuna, 2008).

Dietary strategies should also be viewed from a local or regional perspective as local opportunities can be tapped where these exist, not attempting to force the same strategy where the context is not receptive (Underwood, 2000). A national unified approach is often difficult due to variable conditions and resources, and therefore, local adaptations are necessary requiring local planning where management skills may be lacking. Advocates of gardening cite evidenced that home gardening can be a sustainable strategy to improve food security and household incomes when gardens are well adapted to local agronomic and resource conditions, cultural traditions and preferences (Midmore et al.,1991). Supporters of gardening do not refute the evidence on mis-management of gardening projects. Many supporters of gardening believe that mismanagement and lack of sustainability are largely results of failure to invest the necessary resources in understanding the existing garden system in the context of changing household objectives (Niñez, 1984; Brownrigg, 1985; Midmore et al., 1991). Therefore, "improved" gardens are planned and developed for which the effort and costs for the household often outweigh the benefits, leading to eventual abandonment of the gardens after the project subsidies terminate.

A different approach to food gardening and an understanding of the traditional gardening system is vital for success. If improved gardens are built on the characteristics and objectives of traditional gardens in the region, many resource constraint problems could be anticipated and avoided (FAO, 1997). Even in communities that have not traditionally gardened exploration of nearby communities that do garden can give a more thorough understanding of the constraints (whether climatic, economic or cultural) that have inhibited gardening in the past. Once a good understanding of current gardening practices, constraints and objectives is established, project promoters and households can design appropriate improvements (FAO, 1997).

One of such gaps became clear in a farm worker community in the Orange Free State Province of South Africa, where an OFSP crop was introduced as an agricultural strategy to improve food availability of especially, vitamin A rich vegetables. The aim of this strategy was to increase the production and consumption of vitamin A rich vegetables, thereby, improving dietary diversity. A community garden was established, and community members were trained on the cultivation of OFSP in their own home gardens (including preparation of the soil, irrigation systems, and fencing of the gardens), as well as, food preparation with vitamin A rich vegetables, and finally, demonstrating the outcome with a meal prepared with vitamin A rich vegetables. All the food was prepared utilizing recipes of the community but replacing one of the ingredients with OFSP. The meal consisted of a traditional relish made with OFSP, OFSP served as a cooked vegetable to which butter and sugar was added (to increase availability of the vitamin A) and as part of a stew containing chicken. Stiff maize meal porridge as part of the staple food choice was prepared by the community and served with the meal. The members of the community, who partook in the meal, expressed their appreciation to the acceptability of the taste and texture of OFSP at that time. However, in the following months, they failed to continue cultivating the 
crop despite the introduction, resources and training provided and irrespective of the participation of the local agricultural extension officer that should have provided support and guidance regarding the food gardens and subsequent food utilization. Upon further investigation, it became apparent that they were not utilizing this new food crop appropriately. This was likely due to insufficient education on the crop, its life-cycle and uses. This community concluded that the crop did not survive in the gardens since the leaves were no longer intact, probably due to grazing livestock such as chickens and goats that could access the area occasionally. They assumed that the OFSP roots had also perished and was therefore, not a suitable crop to cultivate in this area. The agricultural extension officer also failed to encourage continuous production of the crop as she also lacked information specific to this crop that she could utilise to educate the community.

Following these findings, it was concluded that reimplementation of training or nutrition education through a food-based approach based on an educational framework designed for the specific community could ensure success in terms of vitamin A intakes, dietary diversity and subsequent household food security. By educating primary food producers, persons responsible for food preparation and persons supervising these activities, the under-utilization or non-utilization of OFSP would not be an obstacle preventing consumption of vitamin A rich foods and dietary diversity in this community. It was also apparent that it is necessary to cover all elements of the food utilization cycle to ensure that nothing is left out in terms of education that will hinder utilization. Here, a simple concept of leaves that did not last lead to non-utilization. Throughout the utilization cycle, there could be many other such obstacles or barriers that could hinder utilization and could in turn impact on food security. The full utilization cycle also impacts the nutritional quality of the crop, thus, there is a dual benefit of designing the educational framework to cover all areas from garden preparation to disposal. This led to the design of the food based approach to successfully re-introduce OFSP to assist in improving the nutritional status of a community firstly by training the trainers responsible for sustainability of the food crops in the rural communities. Achieving sustainable improvements in household nutrition, from a food security angle demands interventions to improve skills, knowledge and community organisation in the areas of food production, post-harvest food management and food utilization (Goodman, 2004).

\section{The role of supervisors and change agents}

What is currently lacking in many interventions is the role of the change agent (FAO, 1997). Training materials designed for Agricultural Extension has been a crucial point to increase the quality of agricultural extension. A study by Hoque and Usami (2008) suggested that providing training program planners with relevant information is crucial to design a more effective training program for the agricultural extension personnel. Another study in Pakistan showed that Agricultural Extension Officers need in-service training to increase their work efficiency (Malik and Khan, 1997). Effectiveness of interventions in many cases depends on staff capacity and attitude, which can be developed through training, visits to successful sites and participatory meetings (Goodman, 2004). In a study conducted in Bangladesh by Helen Keller International, the extension staff was trained in the cultivation of primarily indigenous vitaminrich vegetables using low-cost, low-risk methods for home gardening, and the average weekly per capita vegetable consumption for target households increased from 5.8 to $7.5 \mathrm{~kg}$ (Marsh, 1998).

In another study by Dirorimwe (1998), trainers and extension workers were trained in basic and new concepts in nutrition; the household food security concept and how it is locally perceived; crop, livestock and dietary diversification; management of small animals; common forms of malnutrition, including their detection and monitoring; diarrhoea prevention and management; and community participatory planning and implementation of household food security and nutrition interventions. For these sessions, a training manual was produced through a consultative process to facilitate the training of the extension workers and the communities. These materials served as a reference for follow-up training. The participatory and multi-disciplinary approach to appraising the household food security and nutrition situation was a revelation for extension workers who were often used to working solely on issues in their technical areas, using a top-down delivery of messages or packages of technological options.

Therefore, the aim of this study is to illustrate how a food-based intervention can be designed that integrates both agricultural and nutritional components empowering those involved. It further aims to integrate those unique traits and behaviours that form the basis of behaviour modification or strengthening by training the trainer, in this instance, the agricultural extension officer. A foodbased strategy was designed for a specific community based on an in-depth understanding of the current situation, and was piloted with agricultural extension officers in order to confirm that the designed educational framework and resulting training manual could be utilized to introduce the unknown crop OFSP to any community that will require training for food gardens. The food-based strategy was designed according to the food utilization cycle to ensure that the training provided was systematic and incorporated all the relevant information, and also to ensure that it is conveyed in this sequence to the community in which it is deployed to ensure ease of understanding. 
The focus was on increasing food access (stable local adequate food supply) through encouraging home gardening (production), food availability (sufficient quantities of physically and culturally available food), through food production (by providing tools and knowledge for cultivation), and food utilization (properly used applying nutrition and health knowledge). This was done by educating the community on all elements of the utilization cycle, and with an integrated agricultural component to ensure sustainability.

\section{METHODOLOGY}

\section{Setting and design of the educational framework}

The population that was studied to design the educational framework for introducing a new food crop was a farming community in the Free State Province, on a farm in the Fouriesburg district. The farm (situated between the towns of Clarens and Fouriesburg in the North-eastern part of the province on the R711 main road), covers an area of 1500 ha, producing a variety of commodities like crops (maize, wheat), cattle and sheep for commercial reasons, fruit (apricots, peaches, cherries) and walnuts for use in a guesthouse on the farm. The farm receives an average rainfall of $780 \mathrm{~mm}$ per year. Constraints to farming are either droughts or hail. Labour on the farm is the main source of income and an average income of R600 (\$75) per month per household was calculated at the time of the study. Households consisted of seven members on average, and thus, these people live below the common international poverty line of $\$ 1 /$ day as defined by the World Bank. The male community members were employed as farm workers, whilst some of the females were employed in the guest house operating on the farm. Employment on the farm increased their access to foods available, specifically, to the women working in the guest house, and the variety of their diets. However, the diets of their household members remained less varied.

The educational framework for the food-based intervention was designed by observing and interviewing women living in farmworker households on the farm, and their habits and practices within the household in relation to food access, availability and utilization. Following this, the educational framework was designed and then refined during a workshop with agricultural extension officers, where it was presented, discussed and finalized to be used as an educational tool.

\section{Baseline data}

To design and build an educational framework that adhere to current and existing practices within a community, it was decided to investigate the food utilisation cycle of a known food crop (spinach) and to use this as a basis to develop and introduce a food utilisation cycle for a new food crop (OFSP). This approach is more likely to result in success, and was done in four stages as shown in Figure 2.

Stage 1 - Explore the community at grass-roots level to ensure appropriate data is gathered to build the strategy

Focus group interviews were conducted on current food utilization patterns of spinach (commonly used) and white sweet potatoes (known, but not planted/cultivated; orange cultivar unknown). The women were asked to describe how they currently utilized these crops to understand what actions they take during each phase of utilization, from access to consumption. They were asked to describe which tools and methods were applied, and who participated in all these various activities.

\section{Stage 2 - Observing the utilization cycle of the known food crop}

Subsequently, the women were asked (independently of each other) to demonstrate in their own food preparation area, all the steps they followed in the food provisioning cycle, from acquisition to disposal, to directly observe current practices regarding spinach and sweet potatoes.

Stage 3 - Identifying behaviours, habits and drivers behind utilization of a known food crop, and combining these with the theory to design the educational framework and training manual for a behaviour change approach

Data from the focus group interviews and observations was transcribed and categorized under themes within the food utilization cycle. This information was then used to design an appropriate educational framework and resulting training manual to improve the utilization of an unknown crop (OFSP).

Stage 4 - Testing the strategy by exposing knowledgeable agricultural extension officers to it, and adapting it where necessary

The strategy was tested by exposing agricultural extension officers who are active trainers in communities where food insecurity and malnutrition exist. These officers then provided input on the general approach, the re-application potential of the educational framework and identified areas to improve. This was conducted in a focus group discussion, and the model was explained to the agricultural extension officers who have been involved in the acquisition cycles of various products. Following this stage, the tools were finalized for general use by agricultural extension officers.

\section{RESULTS}

\section{Formulation of the educational framework}

Based on these successful results of an approach where local gardening practices formed an important component of an intervention, and where health, nutrition and agricultural education were integrated, the educational framework for the nutrition intervention was designed according to the steps as outlined in Hoelscher et al. (2002) in the following phases:

\section{Needs assessment}

Knowledge was gathered about the problem and population. This was done by reviewing the data collected through this study as well as, a review of the baseline data collected during other studies. Relevant national surveys were also studied; in this case the NFCS (Labadarios, 2002). For the purpose of this study, spinach was selected as the crop that is most widely consumed in order to conclude on the specific needs of 
Table 1. Findings relating to the utilisation of spinach.

\begin{tabular}{|c|c|c|}
\hline Category & Conclusion & Need to be addressed \\
\hline \multirow[t]{5}{*}{$\begin{array}{l}\text { Food accessing } \\
\text { strategies }\end{array}$} & $\begin{array}{l}\text { The community already cultivates their own crops and have access to land to produce home gardens, although } \\
\text { not optimally. }\end{array}$ & $\begin{array}{l}\text { Home cultivated produce need to be expanded and quantity increased for } \\
\text { sustained incorporation into their diets. }\end{array}$ \\
\hline & Home-gardens are not a new concept. & Hardy crops that can be a food source all year round. \\
\hline & They produce only a limited number of fruits and vegetables and they have a shortage of such during summer. & \\
\hline & $\begin{array}{l}\text { The community relies heavily on the purchasing of foods - as it is their main accessing strategy. They however } \\
\text { access foods through this strategy that they did not cultivate at home. }\end{array}$ & Increased dietary diversity at little or no cost to them. \\
\hline & They have severe monetary constraints. & $\begin{array}{l}\text { Variety of potential uses for crops they are introduced to that could also } \\
\text { potentially reduce their spending on other categories. }\end{array}$ \\
\hline \multirow{3}{*}{$\begin{array}{l}\text { Types of food } \\
\text { accessed }\end{array}$} & They consume a variety of foods, but their weekly variety remains low. & Introducing a crop that satisfies all their needs to increase dietary diversity. \\
\hline & Their diets heavily rely on starchy foods and vegetables. & $\begin{array}{l}\text { OFSP, although a starchy food - has many nutritional advantages - specifically } \\
\text { bio-available vitamin A. }\end{array}$ \\
\hline & $\begin{array}{l}\text { The crops that they do currently produce indicates that they are more likely to produce more hardy crops that } \\
\text { require little human involvement and offers resistance to environmental and weather changes (such as pumpkin, } \\
\text { potatoes and beetroot), or crops that offer versatility in use (such as spinach, tomatoes and cabbage). }\end{array}$ & They need a crop that is hardy and versatile in use. \\
\hline \multirow[t]{2}{*}{ Food production } & $\begin{array}{l}\text { They produce a variety of crops, however spinach is the only crop that is truly produced all year round by some } \\
\text { women, and less often by others (but remains the most produced crop). }\end{array}$ & $\begin{array}{l}\text { The community has a need for a similar crop that fits into their current habits and } \\
\text { practice. }\end{array}$ \\
\hline & They find the crop tasty, versatile and nutritious. & \\
\hline \multirow[t]{3}{*}{ Food gathering } & & The women could access foods from their cultivated gardens. \\
\hline & Ine women currently rely on Wild leaves trom tne surrounaing tield to supplement tne proauction of spinacn. & \multirow{2}{*}{$\begin{array}{l}\text { They would not need to walk long distances in search of wild leaves but could } \\
\text { easily access vegetables on a continuous basis. }\end{array}$} \\
\hline & They did not attempt to increase production from their garden to increase the availability of leafy vegetables. & \\
\hline \multirow{2}{*}{$\begin{array}{l}\text { Food preparation } \\
\text { and consumption }\end{array}$} & The women currently prepare spinach with very little work needed. & They need a crop that can be easily prepared and will be well accepted by all. \\
\hline & It is consumed by the whole family. & \\
\hline \multirow[t]{2}{*}{ Dietary diversity } & The community overall has poor dietary diversity which worsens during the warm summer months. & \multirow{2}{*}{$\begin{array}{l}\text { The introduction of any crop that can be easily home produced. It will be } \\
\text { particularly effective in the warmer months when only tomatoes, cabbage, onions } \\
\text { and potatoes are produced - none of which contain high levels of vitamin A. }\end{array}$} \\
\hline & They consume more starchy foods with lower micronutrient content, including vitamin A. & \\
\hline
\end{tabular}

this community. Key findings are summarised in Table 1.

\section{Objectives}

The objective was to design an intervention through providing both verbal (in the form of a workshop) and written (posters) information that:
1. Increases the nutrition knowledge and awareness of the community as well as the policymakers by providing information on:

(a) The relationship between diet and health (and a varied diet to prevent malnutrition);

(b) The relationship between nutritional and health status, individual productivity and national development; (c) The nutritional needs of the population and of individuals (as per the food based dietary guidelines);

(d) The importance of ensuring the quality and safety of the food supply; and

(e) The causes and consequences of nutritional disorders.

2. Promotes desirable food behaviour and nutritional practices by providing information on: 
(a) The nutritional value of foods (current diet vs. proposed diet including OFSP);

(b) The components of an adequate diet (food groups and portion sizes);

(c) Making appropriate food choices and purchases from available resources;

(d) Hygienic food preparation and handling of food;

(e) Storage, processing and preservation of food; and

(f) Equitable intra-household food distribution according to the nutritional needs of family members.

3. Increases the diversity and quantity of family food supplies by:

(a) Providing information on methods of improving food production;

(b) Crop selection and diversification;

(c) Proper storage, preservation and processing; and

(d) Conservation of nutrients during food preparation; and the prevention of wastage of food.

\section{Developing an educational framework for the nutrition education intervention through intervention mapping}

In order to design effective interventions, the determinants of food choice need to be identified and matched with relevant intervention strategies grounded in a theoretical framework that may most likely produce positive changes in these mediators (Zeidan, 2007). The steps that were followed are:

1. Defining proximal program objectives: Desirable behavioural and environmental outcomes. This program focused on a nutritional message (the benefits of a varied diet), and how the women can utilise OFSP optimally to improve dietary diversity and reduce vitamin A deficiencies. It should encourage and motivate all adult female community members to participate and collaborate in the intended intervention. For families who do not consume OFSP, it should encourage them to include the product in their diet. For families who do consume OFSP or white-fleshed sweet potato, it should encourage them to increase consumption of OFSP to:

(a) Increase dietary diversity;

(b) Increase nutritional knowledge, specifically that of vitamin A rich foods;

(c) Improve and alleviate food insecurity amongst the community;

(d) Build on current knowledge base; and

(e) Enforce any current nutritional knowledge.

2. Delineating intervention methods from theory and translating the methods into practical strategies: In order to translate this into a strategy, the health belief model was applied. This model proposes that several factors affect whether or not people change their behaviour. These include the following; First, evaluation of their own susceptibility to a certain condition, second how serious the consequences of the condition would be, and then, whether a change in behaviour would prevent the condition, and finally, whether the benefits of the change exceed the costs of taking action (Zeidan, 2007).

In this study, vitamin A deficiency was used as the basis to communicate the intervention to the target population in order to encourage OFSP cultivation and utilisation. To assess how this can be communicated and applied, spinach cultivation and utilisation was studied.

\section{The current utilization of spinach}

The findings with regard to the utilization of spinach were incorporated into the educational framework as shown in Figure 1 to reintroduce the unknown crop OFSP. Whilst studying the current habits and practices, reasons for the current approaches were uncovered.

\section{General usage habits and beliefs}

Spinach is consumed often, twice to three times per week. Women mentioned reasons for doing so to be that the crop is nutritious, it assists in preventing disease, it is a convenient food and is also tasty. Thus, OFSP can be introduced by:

(a) Explaining the benefits of the crops in terms of vitamins and minerals;

(b) Explaining the nutrient content by linking them to commonly known and experienced diseases;

(c) Demonstrating techniques and recipes that are easy to prepare with the tools and equipment they have available;

(d) Ensuring that the prepared recipes and products are palatable and tasty.

\section{Procurement}

Spinach is currently continuously harvested from home gardens as needed. This is done as the women realize it is cost saving, and plants it regularly to ensure availability. Based on this, for the introduction of OFSP, the following should be considered:

(a) Emphasize that this crop also requires no monetary input if they continue to cultivate and plant stems;

(b) Ensure it is self-sustaining by providing stems during introduction, and teaching them how to cultivate and plant the stems continuously in an active nursery garden. 


\section{DIETARY DIVERSITY}

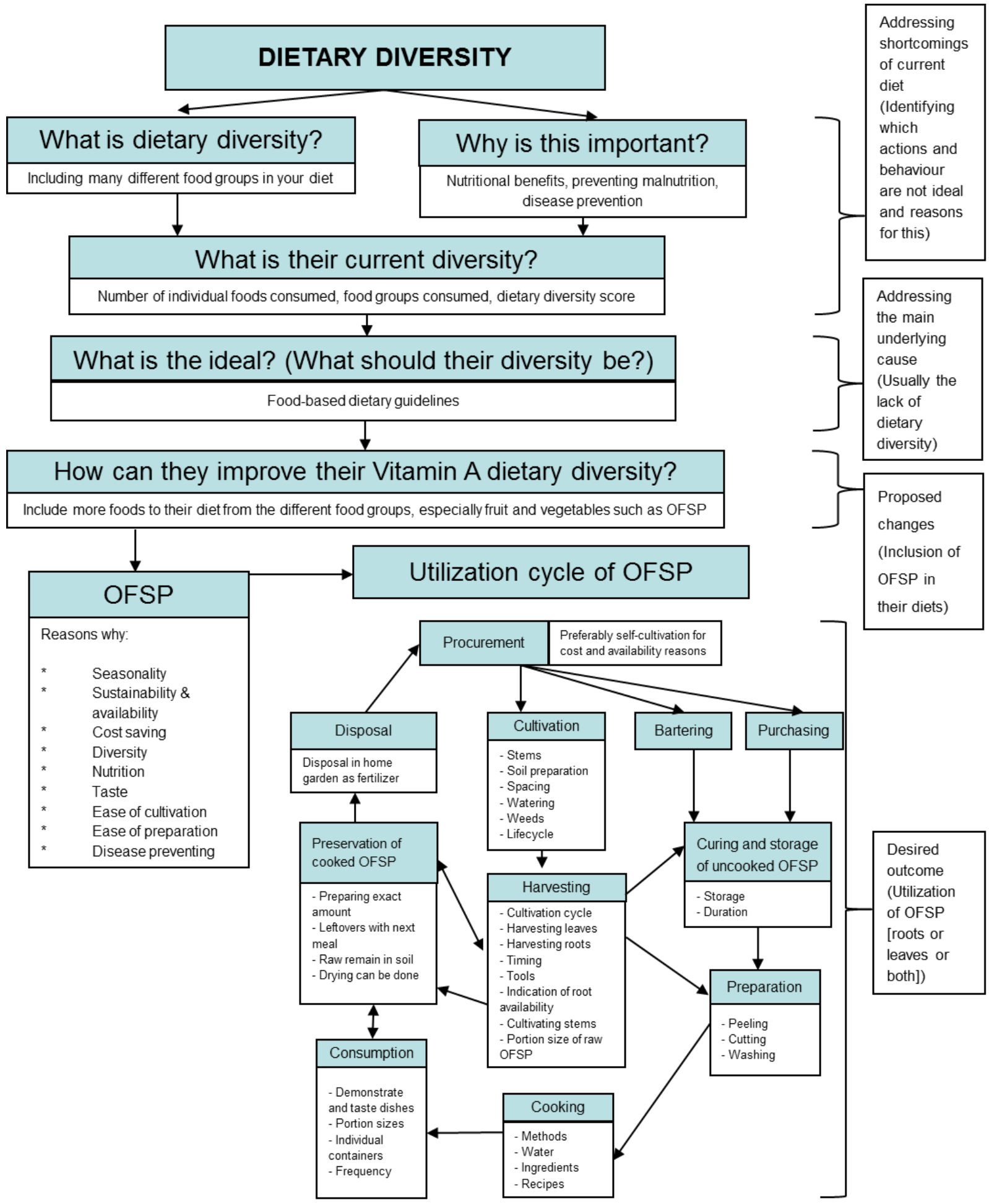

Figure 1. Educational framework for the introduction of SP.

\section{Cultivation}

The main steps of cultivation were covered as outlined in Faber et al. (2006). Spinach is traditionally cultivated from seeds. Before they plant the seeds, they prepare the soil by loosening and watering it. They evenly spaced the seeds, cover them with soil, and water them. Thereafter, they water the spinach daily and remove weeds when 


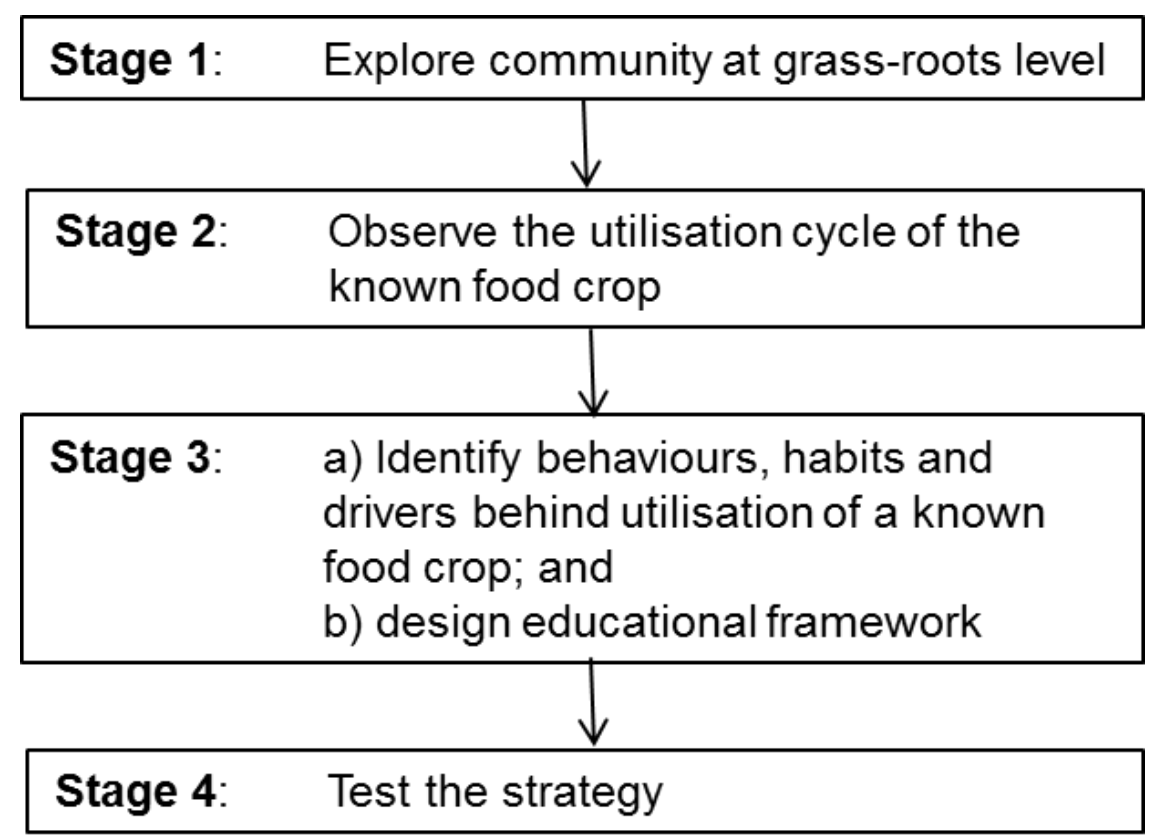

Figure 2. Methodology to design educational framework.

necessary to ensure the plant remains healthy, which in turn will ensure continuous availability. Based on this, when OFSP is introduced, the following should be kept in mind:

(a) Stems should be supplied for first round of planting, and women should be shown how the stems are prepared;

(b) Soil preparation should be discussed, with specific focus on continuous enrichment as they do not currently practice this;

(c) Spacing of plants should be covered as different crops require different spacing;

(d) Watering timing and quantity should also be covered as this is very crop specific;

(e) They should be encouraged to continue removing weeds, however they should be provided with a reference of the leaves of the OFSP plant to ensure it is not mistaken for a weed and removed;

(f) Explain the life cycle of the plant to ensure they understand differences vs. spinach, including time lapse between planting and harvesting, continuous harvesting of both roots and leaves, and removal of the complete plant that would require stems to be re-planted or utilization of the nursery.

\section{Harvesting}

They currently harvest by hand as they have limited tools available and with this method, no tools are required. The crop is harvested as needed for a meal as they have no storage facilities. However, they do practice sun-drying of the leaves if in excess. The women tell when the plant is ready for harvest based on the size of the outer leaves, as they use the leaf size as reference for readiness. Only the outer leaves are removed, as the plant is never fully removed, and it is left to grow new leaves. They know from experience that approximately 30 leaves are sufficient to prepare a relish for a family of five. Based on this, the following would have to be included in the reintroduction strategy:

(a) Women should be educated on the cultivation cycle of the crop to ensure they know when it is ready for harvesting;

(b) Tools should be made available to remove the roots from the soil;

(c) Harvesting of both the OFSP roots and leaves have to be demonstrated, and tools have to be made available for this such as pitch-forks and spades;

(d) Women should be encouraged to also harvest as needed, and to leave the remaining roots in the soil;

(e) Part of the education should focus on the leaves of the crop that can also be prepared as a relish, however that the plant may perish if all the leaves are removed, much like the spinach plant. It is important to understand the growth cycle of the OFSP roots and leaves;

(f) Women should however understand that even if no leaves are visible, roots may remain in the soil;

(g) Women should be educated on the fact that if all roots are removed, some should be kept to cultivate new stems;

(h) Educate women on how many roots and which sizes 
are needed in the raw form to prepare a meal for their family. $0.5 \mathrm{~kg}$ will yield approximately 3 portions (Ceserani et al., 1995).

\section{Preparation}

Spinach leaves were washed in water to remove soil. Afterwards, stems and stalks were removed, being the parts that the women perceive to be less-digestible. It was then cut into small pieces for ease of cooking, consumption and digestion. Thus, the following should be covered in the strategy as outlined by Bennion (1995):

(a) Washing is essential as it removes dirt, pesticide contaminants, microorganisms and mycotoxins;

(b) Spoiled and discolored portions should be trimmed off;

(c) If the skin is removed, it should be scraped, not peeled as the bulk of the nutrients is located underneath the skin;

(d) If the skin is too thick to be scraped, the OFSP should first be cooked before it is peeled to retain nutrients;

(e) Using a sharp knife for cutting also ensures that the minimum amounts of nutrients are lost. A blunt knife used during the preparation of vegetables will cause a decrease in the Vitamin A content;

(f) Washing after the OFSP has been cut will lead to more nutrient losses. The crop should be properly cleaned before cutting;

(g) Soaking the roots in water before cooking will also lead to nutrient losses;

(h) If it is cut, smaller pieces will expose a larger surface area, which will lead to more losses;

(i) The sweet potato should be cooked immediately after cutting or peeling as it can discolour whilst standing.

\section{Cooking}

Women mainly boil the spinach or steam it without water, and they do this for ease of consumption and digestion. They know that spinach generates a lot of liquid whilst steaming, and if the water dries up they would add more water. They then add ingredients such as other vegetables, spices, oil, milk and eggs, and this is to make it tastier and because healthcare professionals have in the past taught them to do this to increase the nutritional value. They cook it for approximately 30 minutes to soften it enough to be used as a relish with maize meal porridge. Based on this the women should be educated on:

(a) Various cooking methods. Roasting in the coals is a very easy method, but will destroy a lot of the nutrient content. Baking in the skin will retain the most vitamins A. It appears to be less stable when heated in a water medium than when heated in a dry state (Bennion, 1995); (b) The amount of water they should add to ensure that they do not add a lot of liquid that will lead to nutrient losses through dissolving or pouring off of the remaining water after cooking (Bennion, 1995);

(c) The benefits of adding additional ingredients (such as oil, butter, sugar, tomatoes) to not only increase the nutritional value of the OFSP, but to also illustrate its versatility;

(d) The approximate cooking time of OFSP to ensure that it is not overcooked that could lead to further nutrient losses;

(e) Cooking temperature is vital. As vitamin A is heat sensitive; any intense or direct heat will lead to increase in losses (Bennion, 1995).

\section{Consumption}

Spinach is served along with other starchy foods such as 'pap' - thick maize meal porridge that forms the basis of most of their meals (staple food). All household members consume spinach as they believe it is tasty, nutritious and aids in disease prevention. The family's meals are served in individual containers, and the women know that the approximate portion size for a spinach relish is 3 tablespoons per adult. This can be reapplied by:

(a) Demonstrating the preparation of various OFSP dishes that can accompany starchy foods such as pap, bread or potato in a sweet or savoury relish;

(b) Encouraging all household members to taste the dishes and to continue consuming the product;

(c) Advising that this dish and any others prepared for the household is served in individual containers to ensure that each family member receives the correct portion size;

(d) Demonstrating the importance of serving the correct portion sizes ( $1 / 2$ cup of cooked OFSP per person) (Whitney and Rolfes, 1999);

(e) Encouraging frequent consumption of the crop.

\section{Preservation}

The women knew of only one preservation method for spinach as they tend to harvest as needed and food very seldom remains after a meal. They would roll the leaves into balls and sun-dry it. However, the resulting product was found to be quite sour and not pleasant tasting. Very few of the households have access to a refrigerator or a freezer; hence, they were in the habit of consuming all that they prepared. Considering this, the following should be incorporated in the strategy:

(a) Women should be encouraged to prepare the exact amount required for a meal for their families as there is no preservation method they can apply with the tools and 
facilities to their disposal;

(b) They should be encouraged to cover any left-over cooked OFSP, and to consume it with the next meal;

(c) Raw OFSP should be left in the soil (Faber et al., 2006), however if it has been harvested/purchased it should be stored in a cool dry place until prepared;

(d) Educate the women on the fact that the crop can however be dried as a method of preservation to ensure access during the few months it cannot be cultivated.

\section{Disposal}

Women disposed of spinach stalks that they removed during preparation in either the garden or regular household waste. Women should be encouraged to dispose of any leftover OFSP in the garden as a form of soil enrichment.

\section{Designing the program and producing the intervention materials: Based on work done by Lytle and Perry (2001) on theory driven healthy behaviour change programs and other planning models, the importance of theory-based and systematic planning of interventions was identified. Planning models help practitioners develop programs step by step using theories to explain and address health problems (Zeidan, 2007). Hence, an educational framework was designed to guide the nutrition intervention strategy. This strategy was based on information gathered on utilisation of spinach and was then applied to design the training manual for the intervention.}

\section{Planning for program adoption and implementation:} Implementation and evaluation of this strategy for use by agricultural extension officers was done concurrently in collaboration with agricultural extension officers, in order to improve the strategy before implementation at community level by these trainers. Agricultural extension officers are active as trainers in the communities as a supporting local gardening and family practise, and are aware of restrictions and limitations as a result of a lack of education, resources and facilities. The strategy was presented to them in a proposed training manual format to illustrate how the strategy would be implemented, and a group work-session facilitated the discussions. The training manual was prepared according to the flow of the educational framework. The overall implementation of the strategy is a problem-solution approach, and was formulated in four phases, and was also explained in these steps:

1. Addressing shortcomings of current diet (Identifying which actions and behaviour are not ideal and reasons for this);
2. Addressing the main underlying cause (Usually the lack of dietary diversity);

3. Proposed changes (Inclusion of OFSP in their diets);

4. Desired outcome (Utilization of OFSP roots or leaves or both).

The session started by discussing the current approach of introducing food gardens within communities. It was discussed that there are in most instances various shortcomings in terms of education provided to the community. It was then proposed that a strategy such as this designed one be applied within their communities. It was explained that this approach is based on an understanding of the habits, practices, knowledge, beliefs and constraints within the community, and to uncover these it is best to study an existing widely consumed crop. The manual encourages the use of group interaction and visual aids, as this makes it easier to involve the illiterate consumer, which is also provided in the manual. It was discussed that the target for implementation would be those household members responsible for food preparation.

The steps in the training manual, the overall approach, the tone of the messages, the visual aids, and the timing of implementation were discussed. They assisted in strengthening the messages based on their knowledge, making it more locally relevant, and including some additional steps or information to ensure that the deployment is tailored to the needs of the community.

\section{Conclusion}

The design of an educational framework for the introduction of an unknown food crop into a farm worker environment was evaluated. The framework was designed by studying the utilization patterns of a known crop by the community and was also shared/tested with agricultural extension officers/trainers to ensure relevance, sufficient content and ease of application within the community. By observing the current strategies, a framework was designed for the unique local situation to ensure maximum acceptance and application by the community. By involving agricultural extension officers/trainers in the process, it was possible to refine the strategy to be used by trainers in any rural community to implement introduction of new food crops to support the community in utilizing the new food crop and to ultimately improve dietary diversity. The successful tool consists of a detailed guideline of how to assess the current access to and utilization of a known food crop, including pre-work that needs to be done on the part of the agricultural extension officer when each aspect of access or step in the utilization cycle is assessed, visual and other aids to apply when interacting with the community to uncover information vital to the design of an educational framework, and a quick reference guide of 
the approach that would/could be used by the agricultural extension officers in their day to day activities.

\section{ACKNOWLEDGEMENTS}

The authors would like to acknowledge the May and Stanley Smith Charitable Trust for funding this research. We also acknowledge Dr. Ciska Green (decd.), Hope Matla and Irene Moopa who conducted parts of the study. We further acknowledge all stakeholders (participants, fieldworkers, and agricultural extension officers) who were responsible for the success of this research, as well as the owner of Orange Farm granting us access. A special thanks to Dr. Sunette Laurie from ARCRoodeplaat for her assistance with the establishment of and community training in the gardens.

\section{REFERENCES}

Allen LH (2008). To what extent can food-based approaches improve micronutrient status? Asia Pac. J. Clin. Nutr. 17 (S1):103-105.

Bennion M (1995). Introductory Foods. Upper Saddle River, New Jersey. Prentice Hall, pp. 284-291.

Brownrigg L (1985). Home gardening in international development. League for International Food Education. Washington DC, USA.

Ceserani V, Kinto R, Foskett D (1995). Practical Cookery. United States. John Wiley \& Sons, pp. 563.

Chakravarty I (2000). Food-based strategies to control vitamin A deficiency. Food Nutr. Bull. 21(2):135-143.

Dirorimwe C (1998). Participatory development of a household food security and nutrition improvement programme in Kano State, Nigeria. Food, Nutrition and Agriculture.http://www.fao.org/docrep/X0051T/X0051t02.htm accessed 27 Dec, 2011.

Faber M, Laurie S, Venter S (2006). Home gardens to address vitamin A deficiency in South Africa: A food-based approach. Pretoria. CDP Printers.

Faber M, Phungula MAS, Venter SL, Dhansay MA, Spinnler BAJ (2002). Home gardens focusing on the production of yellow and darkgreen leafy vegetables increase the serum retinol concentrations of 2-5 year-old children in South Africa. Am. J. Clin. Nutr. 76(5):10481054.

Faber M, van Jaarsveld PJ (2007). The production of provitamin A-rich vegetables in home-gardens as a means of addressing vitamin $A$ deficiency in rural African communities. J. Sci. Food Agric. 87:366377.

FAO (1997). Agriculture, food and nutrition for Africa: a resource book for teachers of agriculture. Human nutrition in the developing world. Food and Agriculture Organization of the United Nations. Food and Nutrition Division, pp. 1-412.

Goodman J (2004). Challenges and Opportunities in Integrating CTC and Food Security Programmes in Malawi (Special Supplement 2).http://fex.ennonline.net/102/5-2-1.aspx accessed 20 Oct 2011.

Heim S, Bauer KW, Stang J, Ireland M (2011). Can a community-based intervention improve the home food environment? Parental Perspectives of the Influence of the Delicious and Nutritious Garden.J. Nutr. Educ. Behav. 43(2):130-134.

Hoelscher DM, Evans A, Parcel G, Kelder S (2002). Designing Effective Nutrition Interventions for Adolescents. J. Am. Diet. Assoc. 102(3):S52-S63.
Hoque MJ, Usami K (2008). Effects of Training on Skill Development of Agricultural Extension Workers in Bangladesh: A Case Study in Four Upazilas(Sub-district) Under Kishoreganj District. J. Soc. Sci. 4(1):2128.

Kuhnlein HV (2000). Finding food sources of vitamin A and provitamin A. Food Nutr. Bull. 21(2):130-134.

Labadarios D (2002). The National Food Consumption Survey (NFCS): Children aged 1-9 years, South Africa, Stellenbosch.

Low JW, Arimond M, Osman N, Cunguara B, Zano F, Tschirley D (2007). A Food-Based Approach Introducing Orange-Fleshed Sweet Potatoes Increased Vitamin A Intake and Serum Retinol Concentrations in Young Children in Rural Mozambique. J. Nutr. pp. 1320-1327.

Lytle LA, Perry C (2001). Applying Research and theory in program planning: An example from a nutrition education intervention. Health Promot. Pract. 2(1):68-80.

Malik TANH, Khan SA (1997). Descriptive Analysis of Biographical and Professional Characteristics of Change Agents in Agriculture: A Study in the Punjab, Pakistan. Pak. J. Agric. Sci. 34:1-4.

Marsh R (1998). Building on traditional gardening to improve household food security. Food, Nutrition and Agriculture.http://www.fao.org/docrep/X0051T/X0051t02.htm accessed 11 Nov, 2011.

Mcaleese JD, Rankin LL (2007). Garden-Based Nutrition Education Affects Fruit and Vegetable Consumption in Sixth-Grade Adolescents. J. Am. Diet. Assoc. 107(4):622-665.

Midmore DJ, Ninez VK, Venkataraman R (1991). Household gardening projects in Asia: past experience and future directions. AVRDC Technical Bulletin No. 19. Shanhua, Taiwan Province of China, AVRDC.

Morris JL, Briggs, M, Zidenberg-Cherr S (2002). Development and Evaluation of a Garden-Enhanced Nutrition Education Curriculum for Elementary School children. J. Child Nutr. Manag. 26(2). http://docs.schoolnutrition.org/newsroom/jenm/02fall/morris/accessed 10 January 2012.

Ninez VK (1984). Household gardens: theoretical considerations on an old survival strategy. International Potato Center.

Underwood (2000). Dietary approaches to the control of Vitamin A deficiency: An introduction and overview. Food Nutr. Bull. 21(2):117123.

Von Braun, J (1993). Urban food insecurity and malnutrition in developing countries: trends, policies and research implications. International Food Policy Research Institute, pp. 1-41.

Whitney EN, Rolfes RR (1999). Understanding nutrition. $9^{\text {th }}$ edition. London. Wadsworth, p. 34.

Zeidan MN (2007). The development, implementation and evaluation of a multi-component nutrition education intervention to promote healthy eating among two Lebanese adolescent samples from contrasting socioeconomic status. PhD thesis. University of Leicester.

Zotor FB, Amuna P (2008). The food multimix concept: new innovative approach to meeting nutritional challenges in Sub-Saharan Africa. Proc. Nutr. Soc. 67:98-104. 